\title{
The collective variables representation of simple fluids from the point of view of statistical field theory*
}

\author{
J.-M.Caillol ${ }^{1}$, O.Patsahan ${ }^{2}$, I.Mryglod ${ }^{2}$ \\ 1 Laboratoire de Physique théorique, CNRS UMR 8627, \\ Bât. 210, Université de Paris-Sud, \\ 91405 Orsay Cedex France \\ 2 Institute for Condensed Matter Physics \\ of the National Academy of Sciences of Ukraine, \\ 1 Svientsitskii Str., 79011 Lviv, Ukraine
}

Received July 18, 2005, in final form October 14, 2005

The collective variable representation (CV) of classical statistical systems such as, for instance, simple liquids has been intensively developed by the Ukrainian school after seminal works by Prof. Ihor Yukhnovskii. The basis and the structure of the CV representation are reexamined here from the point of view of statistical field theory and compared with another exact statistical field representation of liquids based upon a Hubbard-Stratonovich transform. We derive a two-loop expansion for the grand potential and free energy of a simple fluid in both versions of the theory. The results obtained by the two approaches are shown to coincide at each order of the loop expansion. The one-loop results are identical to those obtained within the framework of the random phase approximation of the theory of liquids. However, at the second-loop level, new expressions for pressure and the free energy are obtained, yielding a new type of approximation.

Key words: statistical field theory, loop expansion, collective variables

PACS: $05.20 . J j, 05.70 . \mathrm{Ce}$

\section{Introduction}

Functional methods in modern statistical physics represent one of the most powerful tools for the study of both equilibrium and dynamical properties (see, e.g. [1,2]). In many cases the partition function of simple models (Ising and Heisenberg spins or classical particles in interaction) can be re-expressed as a functional integral after performing a Hubbard-Stratonovich transformation, a simple device proposed in

*This paper is dedicated to Professor Ihor Yukhnovskii on the occasion of his $80^{\text {th }}$ birthday. 
the 50ies $[3,4]$. Nearly at the same time another method - the so-called collective variables $(\mathrm{CV})$ method - that allows us to explicitly construct a functional representation for many-particle interacting systems was developed [5,6] and applied to the description of charged particle systems, in particular, to the calculation of the configurational integral of the Coulomb systems. The idea of this method is based on: (i) the concept of collective coordinates being appropriate for the physics of the system considered (see, for instance, [7]), and (ii) an integral giving an exact functional representation for the configurational Boltzmann factor. Later the CV methods were successfully developed for the description of classical many-particle systems [8] and for the theory of second order phase transitions [9]. The method has a wide range of applications and, to paraphrase Prof. Yukhnovskii in his book [9] "The collective variables is a common name for a class of variables which are specific for each kind of physical system. For magnetic systems CV are variables corresponding to the modes of vibrations of spin moments, for ferroelectrics they are cluster vibrational modes, for systems of charged particles they are associated with the modes of generalized charge vibrations, for binary alloys they are modes of one-particle distribution function, the CV of the liquid-gas system are modes of some deviations from the critical density and so on."

One of the goals of this paper is to reconsider the CV method from the point of view of statistical field theory and to compare the results obtained with those found recently by one of us by means of the KSSHE (Kac-Siegert-Stratonovich-HubbardEdwards) theory [10].

We formulate the method of $\mathrm{CV}$ in real space and consider a one-component continuous model consisting of hard spheres interacting through additive pair potentials. The expression for the functional of the grand partition function is derived and the CV action that depends upon two scalar fields - field $\rho$ connected to the number density of particles and field $\omega$ conjugate to $\rho$ is calculated. We study the correlations between these fields as well as their relations to the density and energy correlations of the fluid. The grand partition function of the model is evaluated in a systematic way using a well-known method of statistical field theory, namely the so-called loop expansion. It consists in functionally expanding the action $\mathcal{H}$ around a saddle point, so that the lowest order (zero loop) approximation defines the meanfield (MF) level of the theory and the first order loop expressions correspond to the random phase approximation (RPA). Recently [10] this technique was applied to the action obtained within the framework of the KSSHE theory. In this paper we perform a two-loop expansion of the pressure and the free energy of the homogeneous fluid which yields a new type of approximation which we plan to test in our future work. We note that another perturbation scheme for the free energy within the framework of the CV method (a group expansion), based on the Gaussian measure, was proposed in the late 70ies [11].

The paper is organized as follows. In section 2, starting from the Hamiltonian, we introduce the two different functional representations of the grand partition function based on the KSSHE and CV methods. Here we also enter several types of statistical field averages that are important in the further part of the paper. In section 3 we 
introduce the CV and KSSHE field correlation functions, establish links between them as well as their relation to the density correlation functions of the fluid. The MF level of the KSSHE and CV field theories is formulated in section 4. Section 5 is devoted to the loop expansion of the grand potential. The pressure and the free energy of the homogeneous fluid are obtained in the two-loop approximation in section 5 . We conclude with some final remarks in section 6 .

\section{Prolegomena}

\subsection{The model}

We consider the case of a simple three dimensional fluid made of identical hard spheres of diameter $\sigma$ with additional isotropic pair interactions $v\left(r_{i j}\right)\left(r_{i j}=\left|x_{i}-x_{j}\right|\right.$, $x_{i}$ is the position of particle " $i$ "). Since $v(r)$ is an arbitrary function of $r$ in the core, i.e. for $r \leqslant \sigma$, we will assume that $v(r)$ has been regularized in such a way that its Fourier transform $\widetilde{v}_{q}$ is a well behaved function of $q$ and that $v(0)$ is a finite quantity. We denote by $\Omega$ the domain of volume $V$ occupied by the molecules of the fluid. The fluid is at equilibrium in the grand canonical (GC) ensemble, $\beta=1 / k_{\mathrm{B}} T$ is the inverse temperature $\left(k_{\mathrm{B}}\right.$ Boltzmann constant), and $\mu$ is the chemical potential. Moreover, the particles are subject to an external potential $\psi(x)$ and we will denote the dimensionless local chemical potential by $\nu(x)=\beta(\mu-\psi(x))$. We will stick to notations usually adopted in standard textbooks devoted to the theory of liquids (see e.g. [12]) and will denote the dimensionless pair interaction by $w(r)=-\beta v(r)$ minus. Quite arbitrarily we will say that the interaction is attractive if the Fourier transform $\widetilde{w}(q)$ is positive for all $q$; in the converse case it will be said to be repulsive.

In a given $\mathrm{GC}$ configuration $\mathcal{C}=\left(N ; x_{1} \ldots x_{N}\right)$ the microscopic density of particles reads

$$
\widehat{\rho}(x \mid \mathcal{C})=\sum_{i=1}^{N} \delta^{(3)}\left(x-x_{i}\right),
$$

and the GC partition function $\Xi[\nu]$ can thus be written as

$$
\begin{aligned}
\Xi[\nu] & =\operatorname{Tr}\left[\exp \left(-\beta V_{\mathrm{HS}}(\mathcal{C})+\frac{1}{2}\langle\widehat{\rho}|w| \widehat{\rho}\rangle+\langle\bar{\nu} \mid \widehat{\rho}\rangle\right)\right], \\
\operatorname{Tr}[\ldots] & =\sum_{N=0}^{\infty} \frac{1}{N !} \int_{\Omega} \mathrm{d} 1 \ldots \mathrm{d} n \ldots,
\end{aligned}
$$

where $i \equiv x_{i}$ and $\mathrm{d} i \equiv \mathrm{d}^{3} x_{i}$. For a given volume $V, \Xi[\nu]$ is a function of $\beta$ and a convex functional of the local chemical potential $\nu(x)$ [13] which we have strengthened by using a bracket. In equation $(2), \exp \left(-\beta V_{\mathrm{HS}}(\mathcal{C})\right)$ denotes the hard sphere contribution to the Boltzmann factor and $\bar{\nu}=\nu+\nu_{\mathrm{S}}$ where $\nu_{\mathrm{S}}=-w(0) / 2$ is $\beta$ times the self-energy of a particle. It follows from our assumptions on $w(r)$ that $\nu_{\mathrm{S}}$ is a finite quantity which depends, however, on the regularization of the potential in the core. In the right hand side of equation (2) we have introduced convenient bracket 
notations

$$
\begin{aligned}
\langle\bar{\nu} \mid \widehat{\rho}\rangle & \equiv \int_{\Omega} \mathrm{d} 1 \bar{\nu}(1) \widehat{\rho}(1), \\
\langle\widehat{\rho}|w| \widehat{\rho}\rangle & \equiv \int_{\Omega} \mathrm{d} 1 \mathrm{~d} 2 \widehat{\rho}(1 \mid \mathcal{C}) w(1,2) \widehat{\rho}(2 \mid \mathcal{C}) .
\end{aligned}
$$

Here we show how to re-express $\Xi[\nu]$ as a functional integral.

\subsection{The Hubbard-Stratonovitch transform}

The Hubbard-Stratonovich transformation [3,4] which was proposed in the 50ies is based on simple properties of Gaussian integrals. Within this framework the GC partition function $\Xi[\nu]$ can be formally re-expressed as a functional integral $[3,4,14-$ 17]. Moreover $\Xi[\nu]$, when re-written in this manner, can be interpreted as the GC partition function of a fluid of bare hard spheres in the presence of an external random Gaussian field $\varphi$ with a covariance given by the pair potential $[10,18,19]$. It will be convenient to distinguish the case of attractive interactions $(\widetilde{w}(q)>0)$ from that of repulsive ones $(\widetilde{w}(q)<0)$.

i) Attractive interactions $(\widetilde{w}(q)>0)$

We start from the well-known property of Gaussian integrals

$$
\begin{aligned}
\exp \left(\frac{1}{2}\langle\widehat{\rho}|w| \widehat{\rho}\rangle\right) & =\frac{\int \mathcal{D} \varphi \exp \left(-\frac{1}{2}\left\langle\varphi\left|w^{-1}\right| \varphi\right\rangle+\langle\widehat{\rho} \mid \varphi\rangle\right)}{\mathcal{N}_{w} \equiv \int \mathcal{D} \varphi \exp \left(-\frac{1}{2}\left\langle\varphi\left|w^{-1}\right| \varphi\right\rangle\right)} \\
& \equiv\langle\exp (\langle\widehat{\rho} \mid \varphi\rangle)\rangle_{w},
\end{aligned}
$$

where $\varphi$ is a real random field and the inverse $w^{-1}$ must be understood in the operator sense, i.e. $\int_{\Omega} \mathrm{d} 3 w(1,3) w^{-1}(3,2)=\delta(1,2)$. The functional integrals which enter the above equation can be given a precise meaning in the case where the domain $\Omega$ is a cube of side $L$ with periodic boundary conditions (PBC) which will be implicitly assumed henceforth. Additional technical details are to be found in the appendix. We now insert equation (5) in the definition (2) of the GC partition function $\Xi[\nu]$ which yields

$$
\begin{aligned}
\Xi[\nu] & =\left\langle\operatorname{Tr}\left[\exp \left(-\beta V_{\mathrm{HS}}(\mathcal{C})+\langle\bar{\nu}+\varphi \mid \widehat{\rho}\rangle\right)\right]\right\rangle_{w} \\
& =\left\langle\Xi_{\mathrm{HS}}[\bar{\nu}+\varphi]\right\rangle_{w} .
\end{aligned}
$$

$\Xi[\nu]$, when re-written in this manner, can thus be re-interpreted as the GC partition function of a fluid of bare hard spheres in the presence of a random Gaussian field $\varphi$ with a covariance given by the pair potential $[10,18,19]$.

ii) Repulsive interactions $(\widetilde{w}(q)<0)$

This time we make use of

$$
\begin{aligned}
\exp \left(-\frac{1}{2}\langle\widehat{\rho}|w| \widehat{\rho}\rangle\right) & =\frac{\int \mathcal{D} \varphi \exp \left(\frac{1}{2}\left\langle\varphi\left|w^{-1}\right| \varphi\right\rangle+\mathrm{i}\langle\widehat{\rho} \mid \varphi\rangle\right)}{\mathcal{N}_{-w} \equiv \int \mathcal{D} \varphi \exp \left(\frac{1}{2}\left\langle\varphi\left|w^{-1}\right| \varphi\right\rangle\right)} \\
& \equiv\langle\exp (\mathrm{i}\langle\widehat{\rho} \mid \varphi\rangle)\rangle_{w}
\end{aligned}
$$


which yields

$$
\Xi[\nu]=\left\langle\Xi_{\mathrm{HS}}[\bar{\nu}+\mathrm{i} \varphi]\right\rangle_{(-w)} .
$$

Note that since $\varphi$ is a real scalar field $\Xi_{\mathrm{HS}}[\bar{\nu}+\mathrm{i} \varphi]$ has to be evaluated for imaginary chemical potentials which may cause some troubles since log $\Xi_{\mathrm{HS}}[\nu]$ has singularities (i.e. branch cuts) in the complex plane [20]. In the repulsive case, the hard core part of the interaction is in fact not compulsory in order to ensure the existence of a thermodynamic limit [21] and the reference system can be chosen as the ideal gas [20,22].

Some comments are in order. Firstly, equations (6) and (8) are easily generalized to the case of mixtures $[19,23]$ or molecular fluids. Secondly, when the pair interaction $w$ is neither attractive nor repulsive, it is necessary to introduce two real scalar fields $\varphi_{+}$and $\varphi_{-}$if some rigor is aimed at [10]. Alternatively, in a more sloppy way, equation (6) can be considered to hold in any cases having in mind that $\varphi$ will be a complex scalar field in the general case. We will thus formally write in all cases

$$
\Xi[\nu]=\mathcal{N}_{w}^{-1} \int \mathcal{D} \varphi \exp \left(-\mathcal{H}_{\mathrm{K}}[\nu, \varphi]\right)
$$

where the action (or effective Hamiltonian) of the KSSHE field theory reads as

$$
\mathcal{H}_{K}[\nu, \varphi]=\frac{1}{2}\left\langle\varphi\left|w^{-1}\right| \varphi\right\rangle-\ln \Xi_{\mathrm{HS}}[\bar{\nu}+\varphi] .
$$

\subsection{The collective variables representation}

Now we briefly introduce the CV representation of $\Xi[\nu]$ and refer the reader to a vast literature for a more detailed presentation (see, e.g. $[6,8,9,24,25])$. The starting point is the formally trivial identity

$$
\exp \left(\frac{1}{2}\langle\widehat{\rho}|w| \widehat{\rho}\rangle\right)=\int \mathcal{D} \rho \delta_{\mathcal{F}}[\rho-\widehat{\rho}] \exp \left(\frac{1}{2}\langle\rho|w| \rho\rangle\right),
$$

where $\delta_{\mathcal{F}}[\rho]$ denotes the functional "delta" [2]. Making use of its functional integral representation (see the appendix)

$$
\delta_{\mathcal{F}}[\lambda] \equiv \int \mathcal{D} \omega \exp (\mathrm{i}\langle\omega \mid \lambda\rangle)
$$

one finds for the GC Boltzmann factor

$$
\exp \left(\frac{1}{2}\langle\widehat{\rho}|w| \widehat{\rho}\rangle\right)=\int \mathcal{D} \rho \mathcal{D} \omega \exp \left(\frac{1}{2}\langle\rho|w| \rho\rangle+\mathrm{i}\langle\omega \mid\{\rho-\widehat{\rho}\}\rangle\right) .
$$

Inserting equation (13) in the definition (2) of the GC partition function $\Xi[\nu]$ one obtains

$$
\Xi[\nu]=\int \mathcal{D} \rho \exp \left(\frac{1}{2}\langle\rho|w| \rho\rangle\right) \mathcal{J}[\rho, \bar{\nu}]
$$


where the Jacobian

$$
\mathcal{J}[\rho, \bar{\nu}]=\int \mathcal{D} \omega \exp (\mathrm{i}\langle\omega \mid \rho\rangle) \Xi_{\mathrm{HS}}[\bar{\nu}-\mathrm{i} \omega]
$$

allows for the passage from the microscopic variables $x_{i}$ to the collective ones $\rho$. We note that $\mathcal{J}[\rho, \bar{\nu}]$ does not depend on the pair interactions $w(1,2)$ but only on the GC partition function of the reference HS system $\Xi_{\mathrm{HS}}[\bar{\nu}]$ which is supposed to be known.

Equation (14) can also easily be recast in the form of a standard statistical field theory, i.e. as

$$
\Xi[\nu]=\int \mathcal{D} \rho \mathcal{D} \omega \exp \left(-\mathcal{H}_{\mathrm{CV}}[\nu, \rho, \omega]\right),
$$

where the action of the CV field theory reads as

$$
\mathcal{H}_{\mathrm{CV}}[\nu, \rho, \omega]=-\frac{1}{2}\langle\rho|w| \rho\rangle-\mathrm{i}\langle\omega \mid \rho\rangle-\ln \Xi_{\mathrm{HS}}[\bar{\nu}-\mathrm{i} \omega]
$$

We stress that $\omega$ and $\rho$ are two real scalar fields and that equations (16) and (17) are valid for repulsive, attractive as well as arbitrary pair interactions. Moreover, with the clever normalization of Wegner [26] for the functional measures there are no unspecified multiplicative constants involved in equation (16) (see the appendix for more details).

The CV transformation is clearly more general than the KSSHE transformation since it can be used for a pair interaction $w(1,2)$ which does not possess an inverse and is easily generalized for n-body interactions $(n>2)$. The equivalence of the $\mathrm{CV}$ and KSSHE representations (9) and (16) of $\Xi[\nu]$ is readily established in the repulsive case $(\widetilde{w}(q)<0)$ by making use of the properties of Gaussian integrals (cf. equation (7)). In the attractive or in the general case we cannot propose a convincing way (i.e. a non-formal one) in order to establish this equivalence.

\subsection{Statistical average}

In the sequel it will be important to distinguish carefully, besides the usual GC average $\langle\mathcal{A}(\mathcal{C})\rangle_{\mathrm{GC}}$ of a dynamic variable $\mathcal{A}(\mathcal{C})$, between two types of statistical field averages. At first the KSSHE averages are defined as

$$
\langle\mathcal{A}[\varphi]\rangle_{\mathrm{K}}=\Xi[\nu]^{-1} \int \mathcal{D} \varphi \mathcal{A}[\varphi] \exp \left(-\mathcal{H}_{\mathrm{K}}[\nu, \varphi]\right)
$$

where $\mathcal{A}[\varphi]$ is some functional of the KSSHE fields $\varphi$ and, secondly the CV averages are defined in a similar way as

$$
\langle\mathcal{A}[\rho, \omega]\rangle_{\mathrm{CV}}=\Xi[\nu]^{-1} \int \mathcal{D} \rho \mathcal{D} \omega \mathcal{A}[\rho, \omega] \exp \left(-\mathcal{H}_{\mathrm{CV}}[\nu, \rho, \omega]\right),
$$

where $\mathcal{A}[\rho, \omega]$ is an arbitrary functional of the two CV fields $\rho$ an $\omega$. 


\section{Correlation functions}

To establish the link between the usual theory of liquids and the two statistical field theories which were introduced in sections (2.2) and (2.3) means to find the relations between density correlation functions on the one hand and field (either KSSHE or CV) correlation functions on the other hand. This is the purpose of the present section.

\subsection{Density correlations}

The ordinary and truncated (or connected) density correlation functions of the fluid will be defined in this paper as $[12,27,28]$

$$
\begin{aligned}
G^{(n)}[\nu](1, \ldots, n) & =\left\langle\prod_{1=1}^{n} \widehat{\rho}\left(x_{i} \mid \mathcal{C}\right)\right\rangle_{\mathrm{GC}}=\frac{1}{\Xi[\nu]} \frac{\delta^{n} \Xi[\nu]}{\delta \nu(1) \ldots \delta \nu(n)}, \\
G^{(n), T}[\nu](1, \ldots, n) & =\frac{\delta^{n} \log \Xi[\nu]}{\delta \nu(1) \ldots \delta \nu(n)} .
\end{aligned}
$$

Our notation emphasizes the fact that the $G^{(n)}$ (connected and not connected) are functionals of the local chemical potential $\nu(x)$ and ordinary functions of the space coordinates $(1, \ldots, n) \equiv\left(x_{1}, \ldots, x_{n}\right)$. We know from the theory of liquids that $[27,28]$

$$
G^{(n), T}[\nu](1, \ldots, n)=G^{(n)}[\nu](1, \ldots, n)-\sum \prod_{m<n} G^{(m), T}[\nu]\left(i_{1}, \ldots, i_{m}\right)
$$

where the sum of products is carried out over all possible partitions of the set $(1, \ldots, n)$ into subsets of cardinal $m<n$. Of course $\rho[\nu](x) \equiv G^{(n=1)}[\nu](x)=$ $G^{(n=1), T}[\nu](x)$ is the local density of the fluid.

It follows from the definition $(20)$ of the $G^{(n)}[\nu](1, \ldots, n)$ that they can be reexpressed as KSSHE or CV statistical averages, i.e.

$$
\begin{aligned}
& G^{(n)}[\nu](1, \ldots, n)=\left\langle G_{\mathrm{HS}}^{(n)}[\bar{\nu}+\varphi](1, \ldots, n)\right\rangle_{\mathrm{K}}, \\
& G^{(n)}[\nu](1, \ldots, n)=\left\langle G_{\mathrm{HS}}^{(n)}[\bar{\nu}-\mathrm{i} \omega](1, \ldots, n)\right\rangle_{\mathrm{CV}} .
\end{aligned}
$$

However, the enlightening of these relations is not of a great use except for the special case $n=1$ which reads explicitly as

$$
\begin{aligned}
\rho[\nu](x) & =\left\langle\rho_{\mathrm{HS}}[\bar{\nu}+\varphi](x)\right\rangle_{\mathrm{K}}, \\
\rho[\nu](x) & =\left\langle\rho_{\mathrm{HS}}[\bar{\nu}-\mathrm{i} \omega](x)\right\rangle_{\mathrm{CV}}
\end{aligned}
$$

where $\rho_{\mathrm{HS}}[\xi](x)$ is the local density of the hard sphere fluid at point $x$ in the presence of the local chemical potential $\xi(x)$. 


\subsection{Field correlations}

The correlation functions of the KSSHE field $\varphi$ and the CV fields $\rho$ and $\omega$ will be defined as

$$
\begin{aligned}
& G_{\varphi}^{(n)}[\nu](1, \ldots, n)=\left\langle\prod_{1=1}^{n} \varphi\left(x_{i}\right)\right\rangle_{\mathrm{K}}, \\
& G_{\rho}^{(n)}[\nu](1, \ldots, n)=\left\langle\prod_{1=1}^{n} \rho\left(x_{i}\right)\right\rangle_{\mathrm{CV}}, \\
& G_{\omega}^{(n)}[\nu](1, \ldots, n)=\left\langle\prod_{1=1}^{n} \omega\left(x_{i}\right)\right\rangle_{\mathrm{CV}},
\end{aligned}
$$

and their truncated counterparts will be defined in the same way as in equation (21). The relations between the field correlation functions (26) and the density correlation functions $G^{(n)}[\nu](1, \ldots, n)$ are easily obtained by introducing ad hoc generating functionals and we quote here only the results referring the reader to the references $[10,29]$ for a detailed discussion.

i) relations between $G_{\varphi}^{(n)}$ and $G^{(n)}$

One has

$$
\begin{aligned}
\langle\varphi(1)\rangle_{\mathrm{K}} & =w\left(1,1^{\prime}\right) \rho[\nu]\left(1^{\prime}\right) \\
G_{\varphi}^{(2), T}[\nu](1,2) & =w(1,2)+w\left(1,1^{\prime}\right) w\left(2,2^{\prime}\right) G^{(2), T}[\nu]\left(1^{\prime}, 2^{\prime}\right) \\
G_{\varphi}^{(n), T}[\nu](1, \ldots, n) & =w\left(1,1^{\prime}\right) \ldots w\left(n, n^{\prime}\right) G^{(n), T}[\nu]\left(1^{\prime}, \ldots, n^{\prime}\right) \text { for } n \geqslant 3,
\end{aligned}
$$

where we have adopted Einstein's convention, i.e. space integration of variables labeled by the same dummy indices is meant. It will be quite enlighting to consider the GC correlations of the microscopic potential

$$
\left.\widehat{V}(x, \mathcal{C}) \equiv \sum_{i=1}^{N} w\left(x, x_{i}\right)=\int_{\Omega} \mathrm{d}^{3} x^{\prime} \widehat{\rho}\left(x^{\prime}, \mathcal{C}\right)\right) w\left(x, x^{\prime}\right)
$$

We readily deduce from equations (27) that

$$
\begin{aligned}
\langle\varphi(1)\rangle_{\mathrm{K}} & =\langle\widehat{V}(1)\rangle_{\mathrm{GC}} \\
G_{\varphi}^{(2), T}[\nu](1,2) & =\langle\widehat{V}(1) \widehat{V}(2)\rangle_{\mathrm{GC}}^{T}+w(1,2), \\
G_{\varphi}^{(n), T}[\nu](1, \ldots, n) & =\langle\widehat{V}(1) \ldots \widehat{V}(n)\rangle_{\mathrm{GC}}^{T} \text { for } n \geqslant 3 .
\end{aligned}
$$

Therefore the KSSHE field $\varphi(x)$ identifies itself "nearly" with the microscopic field $\widehat{V}(x)$ at a neutral point. 
ii) relations between $G_{\rho}^{(n)}, G_{\omega}^{(n)}$ and $G^{(n)}$

One easily finds that

$$
G_{\rho}^{(n), T}[\nu](1, \ldots, n)=G^{(n), T}[\nu](1, \ldots, n),
$$

which was expected and that

$$
G_{\varphi}^{(n), T}[\nu](1, \ldots, n)=(-\mathrm{i})^{n} G_{\omega}^{(n), T}[\nu](1, \ldots, n),
$$

which was suspected. We stress that the above relations are valid for all $n$. In particular, for $n=1$ note that $\langle\rho(x)\rangle_{\mathrm{CV}}=\rho(x)$ and $\langle\omega(x)\rangle_{\mathrm{CV}}=\mathrm{i}\langle\widehat{V}(x)\rangle_{\mathrm{GC}}$. The CV $\rho$ and $\omega$ are thus connected to the density of particles and the microscopic potential respectively. We have therefore the correspondence $\varphi \leftrightarrow-\mathrm{i} \omega$ which will be a guideline in the sequel.

\section{Mean field theory}

Contrary to the situation which prevails in the theory of liquid, the term "meanfield" approximation is defined unambiguously in statistical field theory. It consists in neglecting field fluctuations and in approximating the grand potential (i.e. the logarithm of the partition function) by minus the action at its saddle point [1]. In this section the KSSHE and CV theories will be considered at this level of approximation.

\subsection{KSSHE representation}

The mean-field (MF) or saddle point approximation of the KSSHE theory will therefore be defined by the set of relations $[10,19]$ :

$$
\Xi_{\mathrm{MF}}[\nu]=\exp \left(-\mathcal{H}_{\mathrm{K}}\left[\nu, \varphi_{0}\right]\right)
$$

where, for $\varphi=\varphi_{0}$, the action is stationary, i.e.

$$
\left.\frac{\delta \mathcal{H}_{\mathrm{K}}[\nu, \varphi]}{\delta \varphi}\right|_{\varphi_{0}}=0 .
$$

Replacing the KSSHE action by its expression (10) in equation (33) leads to an implicit equation for $\varphi_{0}$ :

$$
\varphi_{0}(1)=w\left(1,1^{\prime}\right) \rho_{\mathrm{HS}}\left[\bar{\nu}+\varphi_{0}\right]\left(1^{\prime}\right)
$$

which reduces to

$$
\varphi_{0}=\widetilde{w}(0) \rho_{\mathrm{HS}}\left[\bar{\nu}+\varphi_{0}\right]
$$

for a homogeneous system. It follows from the stationary condition (33) that the MF density is given by

$$
\rho_{\mathrm{MF}}[\nu](1)=\frac{\delta \ln \Xi_{\mathrm{MF}}[\nu]}{\delta \nu(1)}=\rho_{\mathrm{HS}}\left[\bar{\nu}+\varphi_{0}\right](1)
$$


and that the MF grand potential reads

$$
\ln \Xi_{\mathrm{MF}}[\nu]=\ln \Xi_{\mathrm{HS}}\left[\bar{\nu}+\varphi_{0}\right]-\frac{1}{2}\left\langle\rho_{\mathrm{MF}}|w| \rho_{\mathrm{MF}}\right\rangle
$$

Moreover, the MF Kohn-Scham free energy defined as the Legendre transform

$$
\beta \mathcal{A}_{\mathrm{MF}}[\rho]=\sup _{\nu}\left\{\langle\rho \mid \nu\rangle-\ln \Xi_{\mathrm{MF}}[\nu]\right\}
$$

is found to be the convex envelop of

$$
\beta \mathcal{A}_{\mathrm{MF}}[\rho]=\beta \mathcal{A}_{\mathrm{HS}}[\rho]-\frac{1}{2}\langle\rho|w| \rho\rangle+\frac{1}{2} \int_{\Omega} \mathrm{d} x w(0) \rho(x) .
$$

It can be shown [10] that $\mathcal{A}_{\mathrm{MF}}[\rho]$ constitutes a rigorous upper bound for the exact free energy $\mathcal{A}[\rho]$ if the interaction is attractive $(\widetilde{w}(q)>0)$ and a lower bound in the converse case $(\widetilde{w}(q)<0)$.

Finally, the pair correlation and vertex (or direct correlation) functions at the zero-loop order which are defined respectively as

$$
\begin{aligned}
G_{\mathrm{MF}}^{(2)}[\nu](1,2) & =\frac{\delta^{2} \ln \Xi_{\mathrm{MF}}[\nu]}{\delta \nu(1) \delta \nu(2)}, \\
C_{\mathrm{MF}}^{(2)}[\rho](1,2) & =-\frac{\delta^{2} \beta \mathcal{A}_{\mathrm{MF}}[\rho]}{\delta \rho(1) \delta \rho(2)},
\end{aligned}
$$

are given by

$$
\begin{aligned}
& G_{\mathrm{MF}}^{(2)}(1,2)=\left(1-w \star G_{\mathrm{HS}}^{(2)}\left[\bar{\nu}+\varphi_{0}\right]\right)^{-1} \star G_{\mathrm{HS}}^{(2)}\left[\bar{\nu}+\varphi_{0}\right](1,2), \\
& C_{\mathrm{MF}}^{(2)}(1,2)=-G_{\mathrm{MF}}^{(2)-1}(1,2)=C_{\mathrm{HS}}^{(2)}(1,2)+w(1,2) .
\end{aligned}
$$

We note that these relations coincide with the old random phase approximation (RPA) of the theory of liquids [12]. Taking advantage of the arbitrariness of $w(r)$ in the core on can choose a regularization potential such that the pair correlation function $g(r)=0$ for $r<\sigma$, then we recover the ORPA (optimized RPA) of the theory of liquids [12]. To be complete let us point out that it follows from equation (27) that the free propagator of the KSSHE field theory is given by

$$
G_{\varphi, \mathrm{MF}}^{(2)}(1,2)=\left(1-w \star G_{\mathrm{HS}}^{(2)}\left[\bar{\nu}+\varphi_{0}\right](1,2)\right)^{-1} \star w(1,2) .
$$

\subsection{CV representation}

An analysis similar to that of section (4.1) can be made in the CV representation. The MF level of the CV field theory will be defined by

$$
\Xi_{\mathrm{MF}}[\nu]=\exp \left(-\mathcal{H}_{\mathrm{CV}}\left[\nu, \rho_{0}, \omega_{0}\right]\right)
$$


where, for $\rho=\rho_{0}$ and $\omega=\omega_{0}$ the $\mathrm{CV}$ action is stationary, i.e.

$$
\left.\frac{\delta \mathcal{H}_{\mathrm{CV}}[\nu, \rho, \omega]}{\delta \rho}\right|_{\left(\rho_{0}, \omega_{0}\right)}=\left.\frac{\delta \mathcal{H}_{\mathrm{CV}}[\nu, \rho, \omega]}{\delta \omega}\right|_{\left(\rho_{0}, \omega_{0}\right)}=0 .
$$

Replacing the CV action by its expression (17) in equation (46) yields a set of two coupled implicit equations for $\rho_{0}$ and $\omega_{0}$ :

$$
\begin{aligned}
& 0=w(1,2) \rho_{0}(2)+i \omega_{0}(1), \\
& 0=\rho_{0}(1)-\rho_{\mathrm{HS}}\left[\bar{\nu}-i \omega_{0}\right](1) .
\end{aligned}
$$

If we define $\varphi_{0}=-\mathrm{i} \omega_{0}$, then the two previous equations can be rewritten

$$
\begin{aligned}
\varphi_{0} & =w(1,2) \rho_{0}(2), \\
\rho_{0}(1) & =\rho_{\mathrm{HS}}\left[\bar{\nu}+\varphi_{0}\right](1),
\end{aligned}
$$

which shows that, as expected, $\varphi_{0}$ coincides with the saddle point of the KSSHE field theory (cf Sec 4.1). Moreover a direct calculation will show that

$$
\ln \Xi_{\mathrm{MF}}[\nu]=-\mathcal{H}_{\mathrm{K}}\left[\nu, \varphi_{0}\right]=-\mathcal{H}_{\mathrm{CV}}\left[\nu, \rho_{0}, \omega_{0}\right]
$$

Therefore the local density, the grand potential, the free energy, the correlation and the direct correlation functions coincide at the MF level in the CV and KSSHE field theories.

\section{Loop expansion}

The loop expansion is a useful tool of statistical field theory [1]. Away from the critical point it yields approximations for the free energy beyond the MF level.

\subsection{Loop expansion of the grand potential}

The loop expansion of the logarithm of the partition function of a scalar field theory can be found in any standard textbook, see e.g. that of Zinn-Justin [1]. In the case of the KSSHE field theory we have just to reproduce the known results. One proceeds as follows. A small dimensionless parameter $\lambda$ is introduced and the loop expansion is obtained by the set of transformations

$$
\begin{aligned}
\varphi & =\varphi_{0}+\lambda^{1 / 2} \chi \\
\ln \Xi[\nu] & =\lambda \ln \left\{\mathcal{N}_{w}^{-1} \int \mathcal{D} \chi \exp \left(-\frac{\mathcal{H}_{\mathrm{K}}[\nu, \varphi]}{\lambda}\right)\right\} \\
& =\ln \Xi^{(0)}[\nu]+\lambda \ln \Xi^{(1)}[\nu]+\lambda^{2} \ln \Xi^{(2)}[\nu]+\mathcal{O}\left(\lambda^{3}\right)
\end{aligned}
$$

where $\varphi_{0}$ is the saddle point of the KSSHE action. The expansion of $\ln \Xi[\nu]$ in powers of $\lambda$ is obtained by performing a cumulant expansion and by making a repeated use of Wick's theorem. At the end of the calculation one set $\lambda=1$. 
In order to obtain a loop expansion of $\ln \Xi$ in the $C V$ representation a little homework is necessary. In this case we are led to introduce the following set of transformations

$$
\begin{aligned}
\rho & =\rho_{0}+\lambda^{1 / 2} \delta \rho, \\
\omega & =\omega_{0}+\lambda^{1 / 2} \delta \omega \\
\ln \Xi[\nu] & =\lambda \ln \left\{\int \mathcal{D} \delta \rho \mathcal{D} \delta \omega \exp \left(-\frac{\mathcal{H}_{\mathrm{CV}}[\nu, \rho, \omega]}{\lambda}\right)\right\},
\end{aligned}
$$

where $\left(\rho_{0}, \omega_{0}\right)$ is the saddle point of the CV action. The form retained in equations $(51)$ and $(52)$ is imposed by the exact relation $\langle\omega\rangle_{\mathrm{CV}}(1)=\mathrm{i} w\left(1,1^{\prime}\right)\langle\rho\rangle_{\mathrm{CV}}\left(1^{\prime}\right)$ (cf. equations (30) and (31) for $n=1$ ). Then one performs a cumulant expansion of (53) and use the appropriate version of Wick's theorem (i.e. for two coupled Gaussian fields $\rho$ and $\omega$ ). The derivation is detailed in reference [29] and will not be reproduced here. One finds that the KSSHE and CV loop expansions of $\ln \Xi[\nu]$ coincide at each order of the expansion (i.e. the $\ln \Xi^{(n)}[\nu]$ are the same) which was expected.

Of course $\Xi^{(0)}[\nu] \equiv \Xi_{\mathrm{MF}}[\nu]$. At the one-loop order one finds $[10,29]$

$$
\Xi^{(1)}[\nu]=\frac{\mathcal{N}_{\Delta_{\varphi_{0}}}}{\mathcal{N}_{w}}=\frac{\int \mathcal{D} \varphi \exp \left(-\frac{1}{2}\left\langle\varphi\left|\Delta_{\varphi_{0}}\right| \varphi\right\rangle\right)}{\int \mathcal{D} \varphi \exp \left(-\frac{1}{2}\langle\varphi|w| \varphi\rangle\right)},
$$

where we have adopted Zinn-Justin's notations for the free propagator [1]

$$
\Delta_{\varphi_{0}}^{-1}(1,2)=w^{-1}(1,2)-G_{\mathrm{HS}}^{(2), T}\left[\bar{\nu}+\varphi_{0}\right](1,2) .
$$

Of course $\Delta_{\varphi_{0}}(1,2)$ coincides with $G_{\varphi, \mathrm{MF}}^{(2)}(1,2)$ as can be seen by comparing equa-

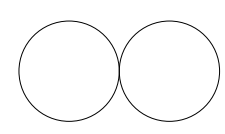

$\mathrm{D}_{\mathrm{a}}$

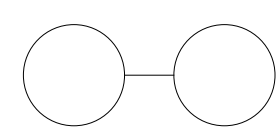

$\mathrm{D}_{\mathrm{b}}$

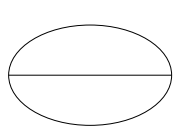

$\mathrm{D}_{\mathrm{c}}$

Figure 1. Diagrams which contribute to $\ln \Xi^{(2)}[\nu] . D_{a}$ and $D_{c}$ are irreducible while $D_{b}$ is reducible.

tions (44) and (55). If $\nu$ is uniform then the system is homogeneous and $\Delta_{\varphi_{0}}(1,2)$ takes on a simple form in Fourier space, i.e.

$$
\widetilde{\Delta}_{\varphi_{0}}(q)=\frac{\widetilde{w}(q)}{1-\widetilde{w}(q) \widetilde{G}_{\mathrm{HS}}^{(2), T}\left[\bar{\nu}+\varphi_{0}\right](q)},
$$

and, in this case, the Gaussian integrals in equation (54) can be performed explicitly (cf the appendix for more details). One finds that

$$
\ln \Xi^{(1)}[\nu]=-\frac{V}{2} \int_{q} \ln \left(1-\widetilde{w}(q) \widetilde{G}_{\mathrm{HS}}^{(2), T}\left[\bar{\nu}+\varphi_{0}\right](q)\right)
$$


The second-loop order contribution $\ln \Xi^{(2)}$ to the grand potential has a complicated expression involving the sum of three diagrams sketched in figure 1

$$
\ln \Xi^{(2)}[\nu]=D_{a}+D_{b}+D_{c} .
$$

More explicitly one has [1]

$$
\begin{aligned}
D_{a}= & \frac{1}{8} \int_{\Omega} \mathrm{d} 1 \ldots \mathrm{d} 4 \Delta_{\varphi_{0}}(1,2) \Delta_{\varphi_{0}}(3,4) G_{\mathrm{HS}}^{(4), T}\left[\bar{\nu}+\varphi_{0}\right](1,2,3,4), \\
D_{b}= & \frac{1}{8} \int_{\Omega} \mathrm{d} 1 \ldots \mathrm{d} 3 \mathrm{~d} 1^{\prime} \ldots \mathrm{d} 3^{\prime} \Delta_{\varphi_{0}}(1,2) \Delta_{\varphi_{0}}\left(1^{\prime}, 2^{\prime}\right) \Delta_{\varphi_{0}}\left(3,3^{\prime}\right) \\
& \times G_{\mathrm{HS}}^{(3), T}\left[\bar{\nu}+\varphi_{0}\right](1,2,3) G_{\mathrm{HS}}^{(3), T}\left[\bar{\nu}+\varphi_{0}\right]\left(1^{\prime}, 2^{\prime}, 3^{\prime}\right), \\
D_{c}= & \frac{1}{12} \int_{\Omega} \mathrm{d} 1 \ldots \mathrm{d} 3 \mathrm{~d} 1^{\prime} \ldots \mathrm{d} 3^{\prime} \Delta_{\varphi_{0}}\left(1,1^{\prime}\right) \Delta_{\varphi_{0}}\left(2,2^{\prime}\right) \Delta_{\varphi_{0}}\left(3,3^{\prime}\right) \\
& \times G_{\mathrm{HS}}^{(3), T}\left[\bar{\nu}+\varphi_{0}\right](1,2,3) G_{\mathrm{HS}}^{(3), T}\left[\bar{\nu}+\varphi_{0}\right]\left(1^{\prime}, 2^{\prime}, 3^{\prime}\right) .
\end{aligned}
$$

As they stand, the above relations are not particularly useful for practical applications (even in the homogeneous case) since they involve the three and four body correlation functions of the reference HS fluid. We will introduce some reasonable approximation in section (5.2) to tackle with this horrible expression. Quite remarkably it has been shown recently that for a symmetric mixture of charged hard spheres $\ln \Xi^{(2)}$ has a much more simple expression which involves only the pair correlation functions of the HS fluid as a consequence of local charge neutrality [19,23].

\subsection{Loop expansion of the pressure and the free energy}

In this section we restrict ourselves to the homogeneous case, therefore $\ln \Xi[\nu]=$ $V \beta P(\nu)$, where $P$ denotes the pressure and $\beta \mathcal{A}[\rho]=V \beta f(\rho)$ where $f$ is the Helmholtz free energy per unit volume. The two-loop expression that we derived for $P$ in section (5.1) is too complicated to be of any practical use since it involves the 3 and 4 body density correlation functions of the HS fluid which are unknown, whereas $G_{\mathrm{HS}}^{(2), T}$ is known approximatively, for instance in the Percus-Yevick (PY) approximation [12]. A simple but coherent approximations for $G_{\mathrm{HS}}^{(3), T}$ and $G_{\mathrm{HS}}^{(4), T}$ will be now proposed.

Recall first that it follows from their definitions [27,28] (see e.g. equations (20)) that the $G_{\mathrm{HS}}^{(n), T}[\nu]$ satisfy the following relations

$$
\frac{\delta}{\delta \nu(n+1)} G_{\mathrm{HS}}^{(n), T}[\nu](1, \ldots, n)=G_{\mathrm{HS}}^{(n+1), T}[\nu](1, \ldots, n, n+1) .
$$

For a homogeneous system (in which case $\nu$ is a constant) one infers from this equation that

$$
\int_{\Omega} \mathrm{d} 1 \ldots \mathrm{d} n G_{\mathrm{HS}}^{(n+1), T}[\nu](1, \ldots, n, n+1)=\frac{\partial^{n}}{\partial \nu^{n}} \rho_{\mathrm{HS}}(\nu) \equiv \rho_{\mathrm{HS}}^{(n)}(\nu)
$$


where $\rho_{\mathrm{HS}}(\nu)$ is the number density of hard spheres at the chemical potential $\nu$. In the rest of the section we will adopt the following approximation

$$
G_{\mathrm{HS}}^{(n), T}[\nu](1, \ldots, n)=\rho_{\mathrm{HS}}^{(n+1)}(\nu) \delta(n, 1) \ldots \delta(2,1) \text { for } n \geqslant 3 .
$$

Note that this hypothesis is coherent with the exact relations (60) and (61). However, we keep the full $G_{\mathrm{HS}}^{(2), T}[\nu](1,2) \equiv G_{\mathrm{HS}}^{(2), T}[\nu]\left(x_{12}\right)$ with the assumption that it is a known function of $x_{12}$ (in the PY approximation for instance). Thus the free propagator $\Delta_{\varphi_{0}}\left(x_{12}\right)$ has a well defined expression as well. It is not difficult to convince oneself that the set of approximations that we have introduced is reasonable as long as the range of the KSSHE field correlation functions is (much) larger than the range of the HS density correlation functions. This will be true if $w$ is a long range pair interaction (near the Kacs limit for instance).

With the hypothesis (62) it is easy to obtain the two-loop order approximation for the pressure. One finds

$$
\begin{aligned}
\beta P(\nu) & =\beta P^{(0)}(\nu)+\lambda \beta P^{(1)}(\nu)+\lambda^{2} \beta P^{(2)}(\nu)+\mathcal{O}\left(\lambda^{3}\right) \\
\beta P^{(0)}(\nu) & =\beta P_{\mathrm{MF}}(\nu)=P_{\mathrm{HS}}\left(\bar{\nu}+\varphi_{0}\right)-\frac{\varphi_{0}^{2}}{2 \widetilde{w}(0)} \\
\beta P^{(1)}(\nu) & =-\frac{1}{2} \int_{q} \ln \left(1-\widetilde{w}(q) \widetilde{G}_{\mathrm{HS}}^{(2), T}\left[\bar{\nu}+\varphi_{0}\right](q)\right) \\
\beta P^{(2)}(\nu) & =\frac{\rho_{0}^{(3)}}{8} \Delta_{\varphi_{0}}^{2}(0)+\frac{\left[\rho_{0}^{(2)}\right]^{2}}{8} \widetilde{\Delta}_{\varphi_{0}}(0) \Delta_{\varphi_{0}}^{2}(0)+\frac{\left[\rho_{0}^{(2)}\right]^{2}}{12} \int \mathrm{d}^{3} x \Delta_{\varphi_{0}}^{3}(x),
\end{aligned}
$$

where $\rho_{0}^{(n)} \equiv \rho_{\mathrm{HS}}^{(n)}\left(\bar{\nu}+\varphi_{0}\right)$ and $\int_{q} \equiv \int \mathrm{d}^{3} q /(2 \pi)^{3}$.

The free energy $\beta f(\rho)$ is obtained as the Legendre transform of $\beta P(\nu)$. The calculation is lengthy and tricky and we must contend ourselves to give the final result (see reference [29] for the complete proof). Setting $\lambda=1$ one finds

$$
\begin{aligned}
\beta f(\rho)= & \beta f_{\mathrm{HS}}(\rho)-\frac{\widetilde{w}(0)}{2} \rho^{2}+\frac{1}{2} \int_{q}\left\{\ln \left(1-\widetilde{w}(q) \widetilde{G}_{\mathrm{HS}, \rho}^{T}(q)\right)+\rho \widetilde{w}(q)\right\} \\
& -\frac{\rho^{(3)}}{8} \Delta_{\rho}^{2}(0)+\frac{1}{8} \Delta_{\rho}^{2}(0) \frac{\left[\rho^{(2)}\right]^{2}}{\rho^{(1)}}-\frac{\left[\rho^{(2)}\right]^{2}}{12} \int \mathrm{d}^{3} x \Delta_{\rho}^{3}(x),
\end{aligned}
$$

where the HS pair correlation function $G_{\mathrm{HS}, \rho}^{T}$ must be evaluated at the density $\rho$ and, similarly,

$$
\widetilde{\Delta}_{\rho}(q)=\frac{\widetilde{w}(q)}{1-\widetilde{G}_{\mathrm{HS}, \rho}^{T}(q) \widetilde{w}(q)}
$$

Finally, in equation (64)

$$
\rho^{(1)}=\frac{1}{\nu_{\mathrm{HS}}^{(1)}(\rho)}, \quad \rho^{(2)}=\frac{\partial \rho_{0}^{(1)}}{\partial \nu}=\frac{-\nu_{\mathrm{HS}}^{(2)}(\rho)}{\left[\nu_{\mathrm{HS}}^{(1)}(\rho)\right]^{3}},
$$




$$
\rho^{(3)}=\frac{\partial \rho_{0}^{(2)}}{\partial \nu}=\frac{3\left[\nu_{\mathrm{HS}}^{(2)}(\rho)\right]^{2}-\nu_{\mathrm{HS}}^{(3)}(\rho) \nu_{\mathrm{HS}}^{(1)}(\rho)}{\left[\nu_{\mathrm{HS}}^{(1)}(\rho)\right]^{5}},
$$

where $\nu_{\mathrm{HS}}^{(n)}(\rho)$ denotes the $n$th derivative of the HS chemical potential with respect to the density (it can be computed within the framework of the PY or CarnahanStarling approximations, for instance [12]). Some remarks are in order.

i) It must be pointed out that, quite unexpectedly, the reducible diagram $D_{b}$ does not have to be cancelled by the Legendre transform. Usually, in statistical field theory it is the case (cf. [1]). The reason is that the chemical potential $\nu$ is not the field conjugate to the order parameter $m=\langle\varphi\rangle_{\mathrm{K}}$ of the KSSHE field theory. However one of us have shown elsewhere $[19,23]$ that for the symmetric mixtures of charged hard spheres only irreducible diagrams contribute to $\beta f^{(2)}$.

ii) The two first terms of equation (64) (i.e. the one-loop order result) supplemented by equation (44) for the pair correlation function are exactly constitutive of the RPA theory of liquid. The full result including the two-loop contribution is a new approximation as far as the authors know.

iii) All the quantities which enter equation (64) can be computed numerically (for instance in the PY approximation). However we stress once again that the validity of equation (64) is restricted to long range pair potentials (for instance $w(r)=\gamma^{3} \Phi(r / \gamma)$ with $\left.\gamma \rightarrow 0\right)$ and we are not aware of the exact results (i.e. obtained by Monte Carlo simulations) which would allow us to test the validity of our expression for the free energy for such kind of fluids.

\section{Conclusion}

Using the CV method we have reconsidered the basic relations of the statistical field theory of simple fluids that follow from this approach. Contrary to the KSSHE theory [10] the corresponding CV action depends on two scalar fields, the field $\rho$ connected to the number density of particles and the field $\omega$ conjugate to $\rho$, connected to the local potential. Explicit relations between field and density correlation functions were obtained.

For a one-component continuous model of fluid, consisting of hard spheres interacting through an attractive pair potential, we have calculated the grand partition function for both versions of statistical field theory using the loop expansion technique. As it was expected, at all the orders of loop expansion considered, both versions of the theory produced indeed the same analytical results. The expressions for the pressure as well as for the free energy were derived at the two-loop level. It yields a new type of approximation and Monte Carlo simulations are wanted to test its validity.

From our analysis of the CV and KSSHE transformations we can also conclude that the former has some important advantages which could be very useful for more 
complicated models of fluids. In particular, it is valid for an arbitrary pair potential (including a pair interaction $w(1,2)$ which does not possess an inverse) and is easily generalized for the case of n-body interparticle interactions with $n>2$.

The statistical field theory of liquids is still in its infancy and many advances including the implementation of Wilson's ideas regarding the renormalization group for the study of gas-liquid critical points are likely to be achieved in the future, this being at least the hope of the authors.

\section{Acknowledgements}

This work was carried out within the framework of the cooperation project between the CNRS and the NASU (ref. CNRS 17110). OP and IM also thank the Fundamental Research State Fund of the Ministry of Education and Science of Ukraine for partial support (Proj. No. 02.07/00303). 


\section{Functional measures and integrals}

In this appendix we give some details concerning functional measures and integrals. Let us consider a real scalar field $\varphi(x)$ defined in a cube $\mathcal{C}_{3}$ of side $L$ and volume $V=L^{3}$. We assume periodic boundary conditions, i.e. we restrict ourselves to the fields which can be written as a Fourier series,

$$
\varphi(x)=\frac{1}{L^{3}} \sum_{q \in \Lambda} \widetilde{\varphi}_{q} \mathrm{e}^{\mathrm{i} q x}
$$

where $\Lambda=(2 \pi / L) \mathbb{Z}^{3}$ is the reciprocal cubic lattice. The reality of $\varphi$ implies that, for $q \neq 0 \widetilde{\varphi}_{q}=\widetilde{\varphi}_{-q}^{\star}$, where the asterisk means complex conjugation. Following Wegner [26] we define the normalized functional measure $\mathcal{D} \varphi$ as

$$
\begin{aligned}
\mathcal{D} \varphi & \equiv \prod_{q \in \Lambda} \frac{\mathrm{d} \widetilde{\varphi}_{q}}{\sqrt{2 \pi V}}, \\
\mathrm{~d} \widetilde{\varphi}_{q} \mathrm{~d} \widetilde{\varphi}_{-q} & =2 \mathrm{dRe} \widetilde{\varphi}_{q} \mathrm{~d} \operatorname{Im} \widetilde{\varphi}_{q} \text { for } q \neq 0 .
\end{aligned}
$$

Equation (6) can thus be conveniently rewritten as

$$
\mathcal{D} \varphi=\frac{\mathrm{d} \varphi_{0}}{\sqrt{2 \pi V}} \prod_{q \in \Lambda^{\star}} \frac{\mathrm{d} \operatorname{Re} \widetilde{\varphi}_{q} \mathrm{~d} \operatorname{Im} \widetilde{\varphi}_{q}}{\pi V}
$$

where the sum in the right hand side runs over only the half $\Lambda^{*}$ of all the vectors of the reciprocal lattice $\Lambda$ (for instance those with $q_{x} \geqslant 0$ ). The normalization constant of equation (6) can thus be explicitly evaluated as

$$
\mathcal{N}_{w}=\int \mathcal{D} \varphi \exp \left(-\frac{1}{2}\left\langle\varphi\left|w^{-1}\right| \varphi\right\rangle\right)=\exp \left(\frac{1}{2} \sum_{q \in \Lambda} \ln \widetilde{w}(q)\right) \sim \exp \left(\frac{V}{2} \int_{q} \ln \widetilde{w}(q)\right)
$$

where $w(1,2)$ is positive and satisfies $w(1,2)=w(2,1) \equiv w\left(x_{12}\right)$ and $(2 \pi)^{3} \int_{q} \equiv$ $\int \mathrm{d}^{3} q$. It is worth noting that in this case we have trivially $\mathcal{N}_{w^{-1}}=1 / \mathcal{N}_{w}$. More generally and with the same hypothesis we have the two useful identities

$$
\begin{aligned}
\langle\exp ((\mathrm{i})\langle\varphi \mid \omega\rangle)\rangle_{w} & \equiv \mathcal{N}_{w}^{-1} \int \mathcal{D} \varphi \exp \left(-\frac{1}{2}\left\langle\varphi\left|w^{-1}\right| \varphi\right\rangle+(\mathrm{i})\langle\varphi \mid \omega\rangle\right) \\
& =\exp \left(+(-) \frac{1}{2}\langle\omega|w| \omega\rangle\right)
\end{aligned}
$$

where $\omega$ is a real scalar field.

Now we define the "functional delta" distribution $\delta_{\mathcal{F}}[\lambda]$ as

$$
\delta_{\mathcal{F}}[\lambda] \equiv \int \mathcal{D} \omega \exp (\mathrm{i}\langle\omega \mid \lambda\rangle)
$$


where both $\omega$ and $\lambda$ are real scalar fields defined on $\mathcal{C}$. Since

$$
\langle\omega \mid \lambda\rangle=\int_{\mathcal{C}} \mathrm{d}^{3} x \omega(x) \lambda(x)=\frac{1}{V} \sum_{q \in \Lambda} \widetilde{\omega}_{-q} \widetilde{\lambda}_{q},
$$

it follows from equation (6) that we have more explicitly

$$
\delta_{\mathcal{F}}[\lambda]=\sqrt{2 \pi V} \delta\left(\widetilde{\lambda}_{0}\right) \prod_{q \in \Lambda^{\star}}\left[\pi V \delta\left(\operatorname{Re} \widetilde{\lambda}_{q}\right) \delta\left(\operatorname{Im} \widetilde{\lambda}_{q}\right)\right] .
$$

Therefore

$$
\int \mathcal{D} \lambda \delta_{\mathcal{F}}[\lambda]=1
$$

and, more generally

$$
\int \mathcal{D} \lambda \mathcal{F}[\lambda] \delta_{\mathcal{F}}\left[\lambda-\lambda_{0}\right]=\mathcal{F}\left[\lambda_{0}\right]
$$

where $\mathcal{F}[\lambda]$ is some arbitrary functional of the field $\lambda(x)$. 


\section{References}

1. Zinn-Justin J. Quantum Field Theory and Critical Phenomena. Clarendon Press, Oxford, 1989.

2. Negele J.W, Orland H. Quantum Many-Particle Systems. Frontiers in Physics, Addison-Wesley, 1988.

3. Hubbard J., Phys. Rev. Lett., 1959, 3, 77.

4. Stratonovich R.L., Sov. Phys. Solid State, 1958, 2, 1824.

5. Zubarev D.N., Dokl. Akad. Nauk SSSR, 1954, 95, 757 (in Russian).

6. Yukhnovskii I.R., Zh. Eksp. Teor. Fiz., 1958, 34, 379 (Sov. Phys. JETP, 34, 263).

7. Bohm D., Pines D., Phys. Rev., 1951, 82, 625.

8. Yukhnovskii I.R., Holovko M.F. Statistical Theory of Classical Equilibrium Systems. Naukova Dumka, Kiev, 1980 (in Russian).

9. Yukhnovskii I.R. Phase Transitions of the Second Order. Collective Variable Methods. World Scientific, Singapore, 1987.

10. Caillol J.-M., Mol. Phys., 2003, 101, 1617.

11. Yukhnovskii I.R. On the statistic theory of condensed systems with long-range and short-range interparticle interactions. Preprint of the Institute for Condensed Matter Physics: ITP-79-133R, Kiev, 1979, 34 p. (in Russian).

12. Hansen J.-P., Mc Donald I.R. Theory of Simple Liquids. Academic Press, London, 1986.

13. Caillol J.-M., J. Phys. A: Math. Gen., 2002, 35, 4189.

14. Kac M., Phys. Fluids, 1959, 2, 8.

15. Siegert A.J.F., Physica, 1960, 26, 530.

16. Hubbard J., Schofield P., Phys. Lett., 1972, 40A, 245.

17. Edwards S.F., Phil. Mag., 1959, 4, 1171.

18. Wiegel F.W., Phys. Rep., 1975, 16C, 57.

19. Caillol J.-M., J. Stat. Phys., 2004, 115, 1461.

20. Caillol J.-M., J. Phys. A: Math. Gen., 2003, 36, 10431.

21. Ruelle D. Statistical Mechanics, Rigorous Results. W.A.Benjamin, New York, 1969.

22. Efimov G.V., Nogovitsin E.A., Physica A, 1996, 234, 506.

23. Caillol J.-M., Mol. Phys., 2005, 103, 1271.

24. Yukhnovskii I.R., Physica A, 1990, 168, 999.

25. Yukhnovskii I.R., Patsahan O.V., J. Stat. Phys., 1995, 81, 647.

26. Wegner F.J. Phase Transitions and Critical Phenomena, Vol. VI, ed. by Domb C. and Green M.S. Academic Press, New York, 1976.

27. Stell G. The Equilibrium Theory of Classical Fluids, eds. Frisch H.L. and Lebowitz J.L.. Benjamin, New York, 1964.

28. Stell G. Phase Transitions and Critical Phenomena, Vol 5b, eds. C. Domb and Lebowitz J.L. Academic Press, 1973.

29. Caillol J.-M., Patsahan O., Mryglod I. Archiv cond-mat/0503213, 2005. 


\title{
Представлення колективних змінних для простих плинів з точки зору статистико-польової теорії
}

\author{
Ж.-М.Кайоль ${ }^{1}$, О.Пацаган ${ }^{2}$, І.Мриглод ${ }^{2}$ \\ 1 Лабораторія теоретичної фізики університету Парі-Сюд, 91405, \\ Орсей, Франція \\ 2 Інститут фізики конденсованих систем НАН України, \\ 79011 Львів, вул. Свенціцького, 1
}

Отримано 18 липня 2005 р., в остаточному вигляді 14 жовтня $2005 \mathrm{p}$.

Започаткований в роботах професора Ігоря Юхновського метод колективних змінних (КЗ) був успішно розвинутий до опису класичних статистичних систем українською школою. В даній роботі основи і структура представлення КЗ для рідин вивчається 3 точки зору статистико-польового підходу і порівнюється 3 іншими точними теоріями, що використовують перетворення Габбарда-Стратоновича. Для випадку простого плину отримано вираз для вільної енергії в обох версіях теорії і показано, що отримані результати співпадають в кожному порядку петлевого розвинення. Результати, отримані в однопетлевому наближенні $€$ ідентичними до отриманих в наближенні хаотичних фаз. Проте, двопетлеве наближення дає новий вираз для тиску і вільної енергії і $€$ новим типом наближення.

Ключові слова: статистико-польова теорія, петлеве розвинення, колективні змінні

PACS: 05.20.Jj, 05.70.Ce 